\title{
Uso de métodos contraceptivos entre acadêmicos da área da saúde
}

\section{Use of contraceptive methods between healthcare academics}

\author{
Eliza Mara das Chagas Paiva ${ }^{1}$, Jenika Ferreira Dias ${ }^{2}$, \\ Anne Pereira Calheiros ${ }^{3}$, Denismar Alves Nogueira ${ }^{4}$, \\ Vânia Regina Bressan ${ }^{5}$, Christianne Alves Pereira Calheiros ${ }^{6}$
}

\begin{abstract}
Resumo
Objetivo: identificar os métodos contraceptivos utilizados por acadêmicos da área de saúde e quais os motivos que influenciam a sua adesão e/ou rejeição. Material e Método: estudo quantitativo, descritivo e transversal realizado com acadêmicos dos cursos de Enfermagem, Fisioterapia e Medicina de uma universidade pública do Sul de Minas Gerais. Para a coleta de dados, utilizou-se um questionário composto por questões referentes a aspectos sociodemográficos, conhecimento geral sobre a sexualidade, riscos de IST/Aids e métodos contraceptivos. Os dados foram analisados por meio da estatística descritiva, utilizando o software SPSS ${ }^{\circledR}$ versão 25.0. Resultados: participaram do estudo 198 acadêmicos. O preservativo masculino constitui o método mais utilizado entre os alunos de ambos os períodos, sendo utilizado por $57,1 \%$ dos alunos. A falta de consciência acerca da importância do uso de contraceptivo na primeira relação sexual foi o maior motivo para a sua baixa adesão $(27,8 \%)$. Todavia, nas relações atuais, identificou-se que ter a relação homoafetiva foi a principal razão para a não adesão (64,3\%). Verifica-se que $67,0 \%$ dos alunos pesquisados afirmam já ter deixado de utilizar o preservativo em alguma relação sexual, sendo a principal justificativa a sua indisponibilidade $(27,0 \%)$. Conclusão: identifica-se ainda barreiras de acesso aos métodos contraceptivos e conhecimento técnico limitado sobre a importância de seu uso. Dessa forma, estratégias de abordagem inovadoras acerca da educação quanto à contracepção devem ser incorporadas e ampliadas de forma efetiva e contínua.

Palavras-chave: Sexualidade. Anticoncepção. Educação superior. Comportamento sexual. Saúde sexual e reprodutiva.
\end{abstract}

\begin{abstract}
Objective: to identify the contraceptive methods used by academics in the health field and what are the reasons that influence their adherence and / or rejection. Material and Method: quantitative, descriptive and cross-sectional study carried out with students from Nursing, Physiotherapy and Medicine courses at a public university in the South of Minas Gerais. For data collection, a questionnaire
\end{abstract}

\footnotetext{
${ }^{1}$ Mestranda no Programa de Pós-graduação em Enfermagem da Universidade Federal de Alfenas (Unifal), Alfenas, Minas Gerais, Brasil. E-mail: elizamdcp@gmail.com

${ }^{2}$ Enfermeira Residente no Programa Multiprofissional em Urgência e Trauma da Pontifícia Universidade Católica de Campinas (PUC-Campinas), Campinas, São Paulo, Brasil.

${ }^{3}$ Graduanda em Medicina na Universidade José do Rosário Vellano (UNIFENAS), Alfenas, Minas Gerais, Brasil.

${ }^{4}$ Doutorado em Estatística e Experimentação Agropecuária pela Universidade Federal de Lavras (UFLA), Lavras, Minas Gerais, Brasil. Professor do Instituto de Ciências Exatas da Universidade Federal de Alfenas, Alfenas, Minas Gerais, Brasil.

${ }^{5}$ Doutorado em Ciências pela Escola Paulista de Enfermagem da Universidade Federal de São Paulo (Unifesp), São Paulo, São Paulo, Brasil. Professora Adjunta da Escola de Enfermagem da Universidade Federal de Alfenas, Alfenas, Minas Gerais, Brasil.

${ }^{6}$ Doutorado em Ciências pela Escola de Enfermagem de Ribeirão Preto da Universidade de São Paulo (EERP-USP), Ribeirão Preto, São Paulo, Brasil. Professora Adjunta da Universidade Federal de Alfenas, Alfenas, Minas Gerais, Brasil.
} 
was used consisting of questions regarding sociodemographic aspects, general knowledge about sexuality, STI / AIDS risks and contraceptive methods. The data were analyzed using descriptive statistics, using the software SPSS ${ }^{\mathrm{TM}}$ version 25.0. Results: 198 students participated in the study. The male condom is the most used method among students of both periods, being used by $57.1 \%$ of students. The lack of awareness about the importance of using contraceptives in the first sexual intercourse was the main reason for their low adherence (27.8\%). However, in current relationships, it was identified that having the same-sex relationship was the main reason for non-adherence $(64.3 \%)$. It appears that $67.0 \%$ of the surveyed students claim to have already stopped using condoms in some sexual relationship, the main reason being their unavailability (27.0\%). Conclusion: access barriers to contraceptive methods and limited technical knowledge about the importance of their use are also identified. Thus, innovative approaches to education about contraception must be incorporated and expanded in an effective and continuous way.

Keywords: Sexuality. Contraception. Higher education. Sexual behavior. Sexual and reproductive health.

\section{Introdução}

A sexualidade diz respeito a algo além do sexo, que se manifesta durante toda a trajetória de vida do ser humano e, mais predominantemente, na juventude, estando em constante desenvolvimento e reestruturação. Todavia, seu exercício nem sempre mostra-se atrelado ao desenvolvimento das dimensões cognitivas e comportamentais, o que desperta preocupação quanto aos riscos de Infecções Sexualmente Transmissíveis (IST) e de gravidez não planejada. ${ }^{(1)}$

Uma fase que pode ser considerada como um fator impulsionante para a expressão da sexualidade é o ingresso na universidade, por expor os acadêmicos a novas experiências, muitas vezes associadas ao uso de bebidas alcoólicas e outras drogas, além de estarem mais vulneráveis a novas vivências antes proibidas ou limitadas pela proximidade familiar, que podem influenciar a prática sexual desprotegida. $^{(2)}$

Ademais, prática de sexo desprotegido é comum entre estudantes universitários e a conscientização sobre as possibilidades de prevenção de IST/Aids e gravidez não planejada ainda não é uma realidade, ${ }^{(3-4)}$ podendo acarretar um aumento da exposição a essas doenças e gravidez não planejada, além de repercussões como o abandono dos estudos e dificuldade de inserção no mercado de trabalho. Desse modo, independentemente do nível de instrução, intervenções e educação sobre métodos contraceptivos devem ser adotadas. ${ }^{(3,5)}$
De acordo com o banco de dados sobre Aids do Ministério da Saúde, desde o ano de 2010 até 2017 foram notificados 153.908 casos desta doença no Brasil. Dentre esses, verifica-se um total de $8.361(5,43 \%)$ e 14.437 (9,38\%) notificações para indivíduos com ensino superior incompleto e completo, respectivamente. ${ }^{(6)}$ Com o advento do HIV/Aids, marcado por um modelo de domínio, passou-se a utilizar o preservativo, no entanto, ainda verifica-se que seu uso é predominante nas relações iniciais, porém, com o tempo de relação é abandonado e passa a ser substituído pela pílula contraceptiva, e quando a relação torna-se ainda mais estável já se usa o dispositivo intrauterino (DIU). Assim, pode-se perceber uma estreita relação entre o tempo de relacionamento e o tipo de método contraceptivo de escolha. ${ }^{(7)}$

Ainda, não se pode negligenciar que a contracepção é permeada por questões de acesso aos métodos contraceptivos e conhecimento. Não obstante, as razões para a sua não utilização ultrapassam essa perspectiva, apresentando-se intimamente articuladas à sexualidade e interação entre o casal, de maneira a influenciar as escolhas sobre como realizar o manejo contraceptivo e a opção por fazê-lo ou não. ${ }^{(7)}$ Nesse contexto, vem à tona a necessidade de se conduzirem discussões acerca da contracepção e sexualidade, a fim de ampliar as políticas públicas sobre o tema. ${ }^{(4,7-8)}$

Ante o exposto, faz-se necessário a realização de estudos nas universidades, locais em que se encontram uma grande parcela de jovens com 
idade em que são notificados elevados números de IST/Aids. Ademais, considerando que as práticas sexuais estão associadas com o conhecimento sobre o assunto, conhecer o comportamento sexual dos acadêmicos da área da saúde é de grande relevância para direcionar as ações de educação em saúde e os currículos de formação acadêmica, uma vez que quando este assunto não é explorado de forma completa entre os acadêmicos pode-se gerar impactos no futuro exercício profissional, podendo acarretar uma insuficiente educação sexual da sociedade. Logo, o presente trabalho objetivou identificar quais são os métodos contraceptivos utilizados por acadêmicos da área de saúde e quais os motivos que influenciam a sua adesão e/ou rejeição.

\section{Material e Método}

Trata-se de um estudo quantitativo, descritivo e transversal, realizado com acadêmicos de ambos os sexos regularmente matriculados nos $1^{\circ}$ e $7^{\circ}$ períodos dos cursos de Enfermagem, Fisioterapia e Medicina de uma universidade pública localizada em um município ao Sul do estado de Minas Gerais, Sudeste brasileiro. A seleção do local do estudo se justifica por tratar-se de uma universidade com um grande número de acadêmicos matriculados em cursos da área da saúde e com base em pesquisas nacionais e internacionais que apontam a manifestação de comportamentos sexuais de risco entre acadêmicos.

Foram definidos os seguintes critérios de inclusão: estar regularmente matriculado no $1^{\circ}$ ou $7^{\circ}$ período dos cursos de Enfermagem, Fisioterapia ou Medicina da universidade supracitada e estar presente no dia da coleta de dados. Optou-se pelos critérios de exclusão: estar ausente da sala de aula no momento da coleta de dados e estar de licença maternidade ou saúde. A amostra foi definida de forma não probabilística, e todos os participantes foram selecionados por conveniência.

Os dados foram coletados por uma das pesquisadoras do estudo, entre os meses de janeiro e março de 2018, em salas de aula, em dias previamente agendados com um docente do curso, sem que prejudicasse o desenvolvimento de sua aula. Utilizou-se para a coleta de dados um questionário( ${ }^{(8)}$ composto por questões abertas e semiabertas, e abordando dados sociodemográficos, conhecimento geral sobre a sexualidade, riscos de IST/Aids e métodos contraceptivos. Após a explicação dos objetivos e procedimentos da pesquisa, os acadêmicos receberam o Termo de Consentimento Livre e Esclarecido (TCLE) para a leitura, e após a assinatura, concordando em participar da pesquisa, o instrumento de coleta de dados foi autoaplicado individualmente, com um tempo gasto para preenchimento de aproximadamente dez minutos. Os dados foram duplamente digitados em planilha Microsoft Excel ${ }^{\circledR}$, versão 2013 e, após validação, realizou-se a análise através de estatística descritiva por meio do programa IBM SPSS ${ }^{\circledR}$, versão 25.0.

Cumpre assinalar que o trabalho foi desenvolvido considerando-se os preceitos éticos contemplados pela Resolução 466/2012, preservando as identidades dos participantes do estudo. Contou-se com a aprovação do Comitê de Ética em Pesquisa com Seres Humanos, conforme Número do Parecer: 1.980.330 (CAAE: 62374216.9.0000.5142).

\section{Resultados}

Participaram do estudo 198 alunos, sendo que $157(79,30 \%)$ afirmaram possuir vida sexual ativa. Verificou-se predominância do sexo feminino $(71,20 \%)$, solteiros $(90,90 \%)$, católicos $(71,20 \%)$, morando com amigos $(54,00 \%)$ e com idade entre 18 e 19 anos (36,90\%). Pouco mais da metade dos participantes não estavam namorando $(53,50 \%)$, não mantinham relacionamento esporádico $(56,60 \%)$ e afirmaram que a sexarca ocorreu entre 16 e 17 anos (42,40\%) e com o namorado (63,90\%).

Em relação ao uso de métodos contraceptivos em primeira relação sexual, no $1^{\circ}$ período 76 alunos $(87,4 \%)$ afirmam utilizá-los, bem como $62(88,6 \%)$ alunos do $7^{\circ}$ período confirmam sua utilização. $\mathrm{O}$ preservativo masculino constitui o método mais utilizado entre os alunos de ambos os períodos, 
sendo utilizado por $45(58,4 \%)$ alunos no $1^{\circ}$ período e $35(55,6 \%)$ no $7^{\circ}$. Seguido, está o uso do preservativo associado à pílula anticoncepcional, mais utilizado no $7^{\circ}$ período $(31,7 \%)$ em relação ao $1^{\circ}$ período $(26,0 \%)$.

Quanto ao motivo para não ter utilizado nenhum método contraceptivo em primeira relação sexual, verificou-se que para os alunos do $1^{\mathrm{o}}$ período essa atitude foi principalmente devido a não dispor do método no momento da relação sexual $(30,00 \%)$ e não ter consciência da necessidade de uso na época em que ocorreu o ato $(30,00 \%)$. Por outro lado, para o $7^{\circ}$ período, $37,50 \%$ dos alunos afirmaram não utilizá-lo devido à influência/escolha e persuasão do parceiro, conforme verifica-se na Tabela 1 .

Tabela 1 - Distribuição numérica e percentual dos participantes da pesquisa de $1^{\circ}$ e $7^{\circ}$ períodos em relação aos aspectos referentes aos métodos contraceptivos utilizados em primeira relação sexual. Alfenas, Minas Gerais, Brasil, 2018.

\begin{tabular}{|c|c|c|c|c|c|c|}
\hline \multirow[t]{2}{*}{ Variáveis relacionadas à primeira relação sexual } & \multicolumn{2}{|c|}{$\begin{array}{c}\mathbf{1}^{\mathbf{0}} \text { período da } \\
\text { graduação } \\
\mathbf{N}=\mathbf{8 7}\end{array}$} & \multicolumn{2}{|c|}{$\begin{array}{c}7^{\circ} \text { período da } \\
\text { graduação } \\
\mathbf{N}=\mathbf{7 0}\end{array}$} & \multicolumn{2}{|c|}{$\begin{array}{c}\text { Total } \\
\mathbf{N}=\mathbf{1 5 7}\end{array}$} \\
\hline & $\mathbf{N}$ & $\%$ & $\mathbf{N}$ & $\%$ & $\mathbf{N}$ & $\%$ \\
\hline \multicolumn{7}{|l|}{$\begin{array}{l}\text { Uso de método contraceptivo } \\
\text { em primeira relação sexual }\end{array}$} \\
\hline Sim & 76 & 87,4 & 62 & 88,6 & 138 & 87,9 \\
\hline Não & 10 & 11,5 & 8 & 11,4 & 18 & 11,5 \\
\hline Não respondeu & 1 & 1,1 & 0 & 0,0 & 1 & 0,6 \\
\hline \multicolumn{7}{|l|}{$\begin{array}{l}\text { Método contraceptivo utilizado } \\
\text { em primeira relação sexual }\end{array}$} \\
\hline Preservativo masculino & 45 & 58,4 & 35 & 55,6 & 80 & 57,1 \\
\hline Injeção anticoncepcional & 1 & 1,3 & 0 & 0,00 & 1 & 0,7 \\
\hline Pílula contraceptiva oral & 8 & 10,4 & 6 & 9,5 & 14 & 10,0 \\
\hline Preservativo feminino & 1 & 1,3 & 0 & 0,00 & 1 & 0,7 \\
\hline Preservativo masculino e coito interrompido & 1 & 1,3 & 0 & 0,00 & 1 & 0,7 \\
\hline Preservativo masculino e pílula anticoncepcional & 20 & 26,0 & 20 & 31,7 & 40 & 28,7 \\
\hline Preservativo masculino e injeção anticoncepcional & 1 & 1,3 & 0 & 0,00 & 1 & 0,7 \\
\hline Preservativo masculino e DIU & 0 & 0,00 & 2 & 3,2 & 2 & 1,4 \\
\hline \multicolumn{7}{|l|}{$\begin{array}{l}\text { Motivo para não utilização de método contraceptivo } \\
\text { em primeira relação sexual }\end{array}$} \\
\hline Não dispor do método no momento & 3 & 30,0 & 0 & 0,0 & 3 & 16,6 \\
\hline $\begin{array}{l}\text { Falta de consciência da importância do uso } \\
\text { na época em que ocorreu a relação }\end{array}$ & 3 & 30,0 & 2 & 25,0 & 5 & 27,8 \\
\hline Não julgar necessário & 1 & 10,0 & 0 & 0,0 & 1 & 5,6 \\
\hline Para que a experiência fosse melhor e mais natural & 1 & 10,0 & 0 & 0,0 & 1 & 5,6 \\
\hline Tratava-se de uma relação homoafetiva & 1 & 10,0 & 2 & 25,0 & 3 & 16,6 \\
\hline Por influência e/ou escolha e/ou persuasão do parceiro & 1 & 10,0 & 3 & 37,5 & 4 & 22,2 \\
\hline Não informado & 0 & 0,0 & 1 & 12,5 & 1 & 5,6 \\
\hline
\end{tabular}

Fonte: Autores 
Evidencia-se que a maioria dos alunos afirmou utilizar algum método contraceptivo atualmente, sendo sua utilização mais predominante no $7^{\circ}$ período $(88,7 \%)$ em relação ao $1^{\circ}(81,6 \%)$. $\mathrm{O}$ preservativo masculino constitui o método mais referido como uso atual pelos acadêmicos, com maior frequência de utilização no $1^{\circ}$ período (40,8\%) em relação ao $7^{\circ}(29,9 \%)$, assim como na sexarca. Todavia, para ambos os períodos, seu uso isoladamente diminuiu de $58,4 \%$ para $40,8 \%$ no $1^{\circ}$ período e de $55,6 \%$ para $29,9 \%$ no $7^{\circ}$ período, se comparada a primeira relação sexual com as atuais.

Com relação ao motivo para não utilizar nenhum método atualmente, no $7^{\circ}$ período, a principal justificativa foi possuir relação homoafetiva (87,5\%). Já no $1^{\circ}, 33,3 \%$ dos alunos afirmaram que esse tipo de relação foi também o motivo para a sua não utilização, bem como o relacionamento de longo tempo e/ou parceiro único $(33,3 \%)$, conforme pode ser observado na Tabela 2.

Tabela 2 - Distribuição numérica e percentual dos participantes da pesquisa de $1^{\circ}$ e $7^{\circ}$ períodos em relação aos aspectos referentes aos métodos contraceptivos utilizados atualmente. Alfenas, Minas Gerais, Brasil, 2018.

\begin{tabular}{|c|c|c|c|c|c|c|}
\hline \multirow[t]{2}{*}{$\begin{array}{l}\text { Variáveis relacionadas aos métodos } \\
\text { contraceptivos utilizados atualmente }\end{array}$} & \multicolumn{2}{|c|}{$\begin{array}{c}\mathbf{1}^{\mathbf{0}} \text { período da } \\
\text { graduação } \\
\mathbf{N}=87\end{array}$} & \multicolumn{2}{|c|}{$\begin{array}{c}7^{\circ} \text { período da } \\
\text { graduação } \\
N=70\end{array}$} & \multicolumn{2}{|c|}{$\begin{array}{c}\text { Total } \\
\mathbf{N}=\mathbf{1 5 7}\end{array}$} \\
\hline & $\mathbf{N}$ & $\%$ & $\mathbf{N}$ & $\%$ & $\mathbf{N}$ & $\%$ \\
\hline \multicolumn{7}{|l|}{ Uso de método contraceptivo atualmente } \\
\hline $\operatorname{Sim}$ & 71 & 81,6 & 62 & 88,7 & 133 & 84,7 \\
\hline Não & 6 & 6,9 & 8 & 11,3 & 14 & 8,9 \\
\hline Não respondeu & 10 & 11,5 & 0 & 0,0 & 10 & 6,4 \\
\hline \multicolumn{7}{|l|}{$\begin{array}{l}\text { Se sim, qual o método contraceptivo } \\
\text { utilizado atualmente }\end{array}$} \\
\hline Preservativo masculino & 29 & 40,8 & 18 & 29,0 & 47 & 35,3 \\
\hline Contraceptivo oral e preservativo masculino & 19 & 26,8 & 24 & 38,8 & 43 & 32,2 \\
\hline Contraceptivo oral & 18 & 25,4 & 13 & 21,0 & 31 & 23,3 \\
\hline Coito interrompido & 1 & 1,4 & 0 & 0,0 & 1 & 0,8 \\
\hline Esterilização cirúrgica & 1 & 1,4 & 0 & 0,0 & 1 & 0,8 \\
\hline DIU & 0 & 0,0 & 3 & 4,8 & 3 & 2,2 \\
\hline Preservativo masculino e pílula de emergência & 1 & 1,4 & 0 & 0,0 & 1 & 0,8 \\
\hline Preservativo masculino e coito interrompido & 1 & 1,4 & 0 & 0,0 & 1 & 0,8 \\
\hline Preservativo masculino e DIU & 0 & 0,0 & 1 & 1,6 & 1 & 0,8 \\
\hline Preservativo masculino e contraceptivo injetável & 0 & 0,0 & 1 & 1,6 & 1 & 0,8 \\
\hline Não respondeu & 1 & 1,4 & 2 & 3,2 & 3 & 2,2 \\
\hline \multicolumn{7}{|l|}{$\begin{array}{l}\text { Motivo para não utilizar nenhum } \\
\text { método atualmente* }\end{array}$} \\
\hline Relacionamento de longo tempo e/ou parceiro (a) único & 2 & 33,3 & 1 & 12,5 & 3 & 21,5 \\
\hline Confiança no (a) parceiro (a) & 1 & 16,7 & 0 & 0,0 & 1 & 7,1 \\
\hline Não julgar necessário & 1 & 16,7 & 0 & 0,0 & 1 & 7,1 \\
\hline Relação homoafetiva & 2 & 33,3 & 7 & 87,5 & 9 & 64,3 \\
\hline
\end{tabular}

Fonte: Autores

*6 alunos do $1^{\circ}$ período e 8 alunos do $7^{\circ}$ período não utilizam métodos contraceptivos. 
Verifica-se que prevalece o uso do preservativo masculino para a maioria dos estudantes pesquisados; no entanto, não fazem uso de maneira rotineira e consistente desse método, uma vez que $67,0 \%$ dos alunos já deixaram de utilizá-lo em alguma relação sexual, sendo esse comportamento discretamente prevalente entre os alunos de $7^{\circ}$ período $(68,2 \%)$ em relação ao $1^{\circ}$ período $(66,0 \%)$.
Dentre os motivos para ter deixado de fazer uso do preservativo masculino, a resposta mais mencionada foi a indisponibilidade do método no momento, o que corresponde a $27,4 \%$ no $1^{\circ}$ período e $26,8 \%$ no $7^{\circ}$ período. Além disso, considerar o uso do preservativo importante constitui a principal justificativa para nunca ter deixado de utilizá-lo, com percentual de $41,2 \%$ no $1^{\circ}$ período e de $35,7 \%$ no $7^{\circ}$ período, conforme evidencia-se na Tabela 3 .

Tabela 3 - Distribuição numérica e percentual dos participantes da pesquisa de $1^{\circ}$ e $7^{\circ}$ períodos em relação aos aspectos referentes ao uso consistente e rotineiro do preservativo masculino. Alfenas, Minas Gerais, Brasil, 2018.

\begin{tabular}{|c|c|c|c|c|c|c|}
\hline \multirow[t]{2}{*}{$\begin{array}{l}\text { Variáveis relacionadas ao uso } \\
\text { de preservativo masculino }\end{array}$} & \multicolumn{2}{|c|}{$\begin{array}{c}\mathbf{1}^{\mathbf{0}} \text { período da } \\
\text { graduação } \\
\mathrm{N}=\mathbf{5 0}\end{array}$} & \multicolumn{2}{|c|}{$\begin{array}{c}7^{\circ} \text { período da } \\
\text { graduação } \\
\mathrm{N}=44^{*}\end{array}$} & \multicolumn{2}{|c|}{$\begin{array}{c}\text { Total } \\
\mathbf{N}=94^{*}\end{array}$} \\
\hline & $\mathbf{N}$ & $\%$ & $\mathbf{N}$ & $\%$ & $\mathbf{N}$ & $\%$ \\
\hline \multicolumn{7}{|l|}{ Se faz uso de preservativo, já deixou de usar* } \\
\hline Sim & 33 & 66,0 & 30 & 68,2 & 63 & 67,0 \\
\hline Não & 17 & 34,0 & 14 & 31,8 & 31 & 33,0 \\
\hline Subtotal & 50 & 100,0 & 44 & 100,0 & 94 & 100,0 \\
\hline \multicolumn{7}{|l|}{ Motivo para ter deixado de fazer uso do preservativo } \\
\hline Aumentar o contato sexual e sentir mais prazer & 7 & 15,9 & 2 & 6,7 & 9 & 12,2 \\
\hline Indisponibilidade do preservativo no momento & 12 & 27,4 & 8 & 26,8 & 20 & 27,0 \\
\hline Não julgar necessário, por uso de contraceptivo oral & 3 & 6,8 & 1 & 3,3 & 4 & 5,4 \\
\hline $\begin{array}{l}\text { Confiança no (a) parceiro (a) / conhecer o (a) } \\
\text { parceiro (a) / relacionamento de longo tempo }\end{array}$ & 3 & 6,8 & 10 & 33,3 & 13 & 17,6 \\
\hline Pretensão em utilizar contraceptivo de emergência & 1 & 2,3 & 0 & 0,0 & 1 & 1,4 \\
\hline Estar empolgado (a) com o prazer no momento & 6 & 13,6 & 3 & 10,0 & 9 & 12,2 \\
\hline Não considerar o método como prático & 6 & 13,6 & 0 & 0,0 & 6 & 8,1 \\
\hline Estar alcoolizado (a) & 2 & 4,5 & 1 & 3,3 & 3 & 4,1 \\
\hline Para não ter desconforto & 1 & 2,3 & 1 & 3,3 & 2 & 2,6 \\
\hline Esquecimento & 2 & 4,5 & 3 & 10,0 & 5 & 6,8 \\
\hline Persuasão e/ou escolha do parceiro (a) & 1 & 2,3 & 1 & 3,3 & 2 & 2,6 \\
\hline Subtotal & $44 * *$ & 100,0 & 30 & 100,0 & 74 & 100,0 \\
\hline \multicolumn{7}{|l|}{$\begin{array}{l}\text { Motivo para nunca ter deixado de fazer uso } \\
\text { do preservativo }\end{array}$} \\
\hline Consideração da importância do uso & 7 & 41,2 & 5 & 35,7 & 12 & 38,7 \\
\hline Para prevenção de IST & 4 & 23,5 & 2 & 14,3 & 6 & 19,4 \\
\hline Para prevenção de IST e de gravidez & 6 & 35,3 & 5 & 35,7 & 11 & 35,5 \\
\hline Para prevenção de gravidez & 0 & 0,0 & 2 & 14,3 & 2 & 6,4 \\
\hline Subtotal & 17 & 100,0 & 14 & 100,0 & 31 & 100,0 \\
\hline
\end{tabular}

Fonte: Autores

Nota: *Número de alunos que responderam a questão. **44 alunos responderam a questão, apesar de apenas 33 alegarem ter deixado de utilizar preservativo em alguma relação sexual. 


\section{Discussão}

A partir dos resultados, verifica-se que o presente estudo demonstrou a utilização do preservativo masculino como o meio de maior proteção anticoncepcional no início da vida sexual dos alunos pesquisados; em seguida, este vem associado à pílula anticoncepcional, a qual constitui a segunda opção de contracepção pelos alunos; entretanto, entre os alunos do $7^{\circ}$ período, nas relações atuais, o uso do preservativo em associação à pílula anticoncepcional é mais frequente, demonstrando que o tempo de graduação pode contribuir para a adesão à dupla proteção. Ao mesmo tempo, identifica-se que apesar de os alunos alegarem prevenirem-se contra a gravidez, até mesmo com dupla proteção, verifica-se que entre o período do início da vida sexual para o momento atual, diminuiu-se a utilização do preservativo masculino, deixando os jovens mais desprotegidos para as Infecções Sexualmente Transmissíveis.

Os presentes achados entram em consonância com dados de um estudo realizado com 566 alunos da Universidade de Sevilha, na Espanha, evidenciando que $86,9 \%$ dos participantes utilizaram método contraceptivo na primeira relação sexual, sendo o preservativo o mais utilizado $(90,6 \%)$, de forma ainda mais frequente que no presente trabalho. ${ }^{(4)}$

Observou-se que os principais motivos que influenciaram o não uso do preservativo na primeira relação sexual por alunos do $1^{\circ}$ período foram não dispor do método no momento da relação sexual e não ter consciência da necessidade de uso na época em que ocorreu. Esses achados remetem a falha da efetividade das políticas de educação sexual sobre a importância do uso do preservativo, bem como os obstáculos de acesso do método, de forma a garantir seu uso. $^{(4)}$

Já para os alunos do $7^{\circ}$ período, aspecto a ser ressaltado é o caráter de convencimento que o parceiro exerce para a não utilização do preservativo, de maneira semelhante aos resultados de outros trabalhos que observaram que a confiança no companheiro, o desejo de agradá-lo e a dificuldade de negociação do uso do preservativo exerce influência sobre o uso do preservativo. $^{(9-10)}$ Um estudo realizado no Rio Grande do Sul com 1.215 universitários constatou que não ter companheiro aumentou em 3,36\% o uso do preservativo. ${ }^{(11)}$

A influência do companheiro no uso do preservativo pode ser explicada pelas peculiaridades dos relacionamentos, como a dificuldade de negociação, preferência por outros métodos, crença da não necessidade de sua utilização com parceiros estáveis e a falsa concepção de que seu uso submete-se a casos extraconjugais, além de que, muitas vezes as ações de educação sexual não contemplam grupos que se encontram em relacionamentos de longa data. Reconhecendo a diversidade presente no ambiente universitário, ações nesse contexto devem ser implantadas como forma de obter melhorias para a saúde da população. $^{(11)}$

Observou-se que o preservativo é o principal método utilizado pelos alunos, tanto na primeira relação sexual como nas demais, independentemente do período o qual está sendo cursado. No entanto, nota-se que seu uso é menos frequente nas relações sexuais habituais se comparado com a primeira, o que corrobora os resultados de outro estudo, em que na sexarca a sua utilização foi significativamente maior do que a verificada em última atividade sexual. Os autores relatam ainda que entre os fatores que influenciam em deixar de utilizá-lo com o passar dos anos, pode-se citar o foco na prevenção de gravidez e não mais em IST/Aids, sobretudo em relacionamentos estáveis, além da preferência por outros métodos contraceptivos, como a pílula anticoncepcional e a crença de que o uso do preservativo submete-se a casos extraconjugais. ${ }^{(11)}$

Estudos internacionais também confirmam a tendência encontrada no presente trabalho no que tange ao uso do preservativo e da pílula anticoncepcional como principais métodos utilizados por estudantes de graduação. $^{(4)}$ Não obstante, apesar da alta aceitabilidade, verificam-se descuidos quanto ao uso de maneira adequada das pílulas anticoncepcionais, dificultando a eficiência 
do método e comprometendo a prevenção de gravidez. Além disso, os conhecimentos acerca dos efeitos colaterais e benefícios do método mostramse insuficientes, ainda que no tocante da esfera de alunos da área de saúde. ${ }^{(12)}$

Os motivos para não utilizar nenhum método contraceptivo nas relações sexuais atuais, como possuir relação homoafetiva, indica a falta de conscientização e conhecimento sobre os riscos da relação sexual desprotegida entre um dos grupos de maior vulnerabilidade a IST/Aids e aponta a importância de conduzir ações educativas estratégicas, principalmente entre alunos de períodos avançados da graduação, ao passo que entre os alunos do $7^{\circ}$ período, $87,5 \%$ argumentaram ser esse o principal motivo para não fazer uso do preservativo.

Outro aspecto investigado foi em relação ao uso consistente e rotineiro do preservativo, em que se pode observar que entre os alunos que utilizam esse método, grande parte já deixou de utilizá-lo em alguma relação sexual $\left(66,0 \%\right.$ e $68,2 \%$ nos $1^{\circ}$ e $7^{\circ}$ períodos). Entretanto, a desproteção quanto ao uso do preservativo é ainda mais evidente em uma pesquisa realizada no Paraná com 184 alunos universitários, a qual observou que $85,8 \%$ já deixaram de utilizá-lo em alguma relação sexual e $6,7 \%$ referiram nunca tê-lo utilizado. ${ }^{(10)}$

A indisponibilidade do método durante o ato sexual como motivo para não utilizá-lo é sustentada nos achados de outro estudo realizado com 498 alunos universitários na Nigéria. Por conseguinte, o uso do preservativo torna-se mais consistente em regiões que dispõem de mais ações intervencionistas de redução dos riscos e de sua distribuição, o que justifica a distribuição de preservativos nas universidades como uma forma de promover seu uso. ${ }^{(13)}$

No que se refere aos motivos para deixar de fazer uso e aqueles que contribuíram para o uso consistente encontrados neste estudo, percebe-se que são condutas que podem ser modificadas a partir da conscientização, quanto atitudes seguras. Contradição digna de nota pode ser observada em outro estudo, o qual notou que a atividade sexual com parceiro fixo constituiu um fator associado a uma maior probabilidade de usar de forma consistente o preservativo. Os autores alegam a possibilidade de que indivíduos que possuem relações sexuais com parceiros esporádicos podem optar por utilizar o preservativo, ao passo que nas relações fixas poderiam deixar de utilizá-lo. ${ }^{(13)}$ Assim, nesse contexto não basta apenas a disponibilização do preservativo, mas também são necessárias ações de educação e conscientização sobre a importância de atitudes sexuais adequadas em todo contato sexual. ${ }^{(10)}$

Cumpre assinalar que a escolha dos métodos contraceptivos e a continuidade ou descontinuidade de seu uso não diz respeito apenas a aspectos individuais de quem o utiliza, mas envolve o contexto sociocultural específico, atendendo às suas particularidades e necessidades primordiais de cada momento de sua vida. ${ }^{(14)}$ Assim sendo, sua aceitabilidade é também influenciada por sua eficácia, facilidade de obtenção, características estéticas, capacidade de ser usado secretamente e de proporcionar e manter o prazer sexual. ${ }^{(15)}$

Haja vista que algumas das barreiras relacionadas ao não uso do preservativo são relacionadas com a possibilidade de diminuição da sensibilidade, da lubrificação e do prazer, ou mesmo a dor decorrente de seu uso, faz-se necessário que a disseminação de conhecimento nesse contexto seja centrada na pessoa e em seu bem-estar na relação, bem como as formas de usar o preservativo baseando-se em uma melhor experiência sexual, considerando-se a ampla disponibilidade de tamanhos, texturas, lubrificação e tipos desse método que estão disponíveis no mercado. ${ }^{(15)}$

Diante das vulnerabilidades identificadas, destaca-se a importância da continuidade do desenvolvimento de estudos que investiguem as demandas e lacunas sobre a contracepção em estudantes universitários. Além disso, torna-se necessário estender as investigações para demais áreas do conhecimento, haja vista que é esperado que alunos das Ciências da Saúde demonstrem comportamentos sexuais mais saudáveis comparados com os demais campos do saber. ${ }^{(4)}$ 
Como limitações do estudo, aponta-se a investigação de um tema que mesmo na atualidade é constituído por tabus e preconceitos, o que pode ter influenciado a resposta dos participantes do estudo. Portanto, sugerem-se novas pesquisas longitudinais que analisem as atitudes dos mesmos participantes ao longo da graduação e que incorporem novos métodos de coleta de dados que assegurem mais privacidade durante a aplicação do questionário.

\section{Conclusão}

Este estudo contribui para o conhecimento acerca dos métodos contraceptivos utilizados por acadêmicos da área de saúde e aponta quais os motivos influenciam a sua adesão e/ou rejeição. Os achados revelaram que o uso de métodos contraceptivos de forma consistente ainda não é uma realidade entre esses alunos, o que se deve, principalmente, à indisponibilidade do preservativo. Dessa forma, há a necessidade da realização de ações de educação em saúde direcionadas e elaboração de políticas públicas para atuação frente a essa temática.

Ainda, os dados apresentados apontam a necessidade da realização de estratégias de abordagem inovadoras, efetivas e permanentes para os acadêmicos da área da saúde, considerando que a universidade constitui um espaço propício para o contato direto e contínuo.

\section{Referências}

1 Teixeira RC, Maria ESC, Silva FJ, Kietzer KS, Nunes EFC, Andrade FSSD, et al. Uso de preservativos por alunos de cursos de saúde em uma universidade pública. Semina: Ciênc Biol Saúde [Internet]. 2018 [citado 2018 nov 16]; 39(1):85-90. Disponível em: http:// www.uel.br/seer/index.php/seminabio/article/ view/30588/23789

2 Borges MR, Silveira RE, Santos AS, Lippi UG. Comportamento sexual de ingressantes universitários. R. Pesq.: Cuid. Fundam. Online [Internet]. 2015 [citado 2018 maio 19]; 7(2).
Disponível em: http://dx.doi.org/10.9789/21755361.2015.v7i2.2505-2515

3 Ajayi AI, Nwokocha EE, Adeniyi OV, Goon DT, Akpan W. Unplanned pregnancy-risks and use of emergency contraception: a survey of two Nigerian Universities. BMC Health Serv. Res. [Internet]. 2017 [citado 2019 Jan 25]; 17(10):382-90. Disponível em: https://www. ncbi.nlm.nih.gov/pmc/articles/PMC5455101/ pdf/12913_2017_Article_2328.pdf

4 Leon-Larios F, Macías-Seda J. Factors related to healthy sexual and contraceptive behaviors in undergraduate students at university of Seville: a crosssectional study. Reprod Health. [Internet]. 2017 [citado 2019 out 14]; 14(179):1-9. Disponível em: https:/www.ncbi.nlm.nih.gov/ pmc/articles/PMC5747274/pdf/12978_2017_ Article_444.pdf

5 Dias EG, Jorge SA, Alves BVC. Alves JCS. Conhecimento e comportamento dos adolescentes de uma escola pública sobre sexualidade e métodos contraceptivos. Rev. Baiana Saúde Pública [Internet]. 2017 [citado 14 ago 2019]; 41(1): 120-30. Disponível em:http://rbsp.sesab.ba.gov.br/index.php/rbsp/ article/view/2408/2183

6 Ministério da Saúde (BR). Dados de aids no Brasil (Banco de dados). Disponível em: http:// www2.aids.gov.br/final/dados/dados_aids.asp [citado 2019 ago 24].

7 Cabral CS. Articulações entre contracepção, sexualidade e relações de gênero. Saude Soc. [Internet].2017 [citado2019 ago 24];26(4):1093104. Disponível em: http://www.scielo.br/ scielo.php?script $=$ sci_arttext\&pid $=$ S010412902017000401093\&lng=en. http://dx.doi. org/10.1590/s0104-12902017000001

8 Alencar RA. Pesquisa-ação sobre sexualidade e vulnerabilidade às IST/aids com alunos de graduação em enfermagem [dissertação]. Ribeirão Preto (SP): Universidade de São Paulo; 2007.

9 Cordeiro JKR, Santos MM, Sales LKO, Morais IF; Dutra GRSF. Adolescentes escolares acerca das DST/AIDS: quando o conhecimento não acompanha as práticas seguras. Rev. Enferm. 
UFPE online. [Internet]. 2017 [citado 2019 ago 24]; 11(Supl. 7):2888-96. Disponível em: https://periodicos.ufpe.br/revistas/ revistaenfermagem/article/view/9014/19196

10 Tomiyoshi MM, Vivan Filho AS, Dias FGF. Avaliação do perfil epidemiológico e comportamental de estudantes de ensino superior em centro universitário privado de Maringá em relação à sexualidade e práticas de risco para doenças sexualmente transmissíveis. Rev. Uningá [Internet]. 2016 [citado 2019 out 4]; 47(1):24-9. Disponível em: http:// revista.uninga.br/index.php/uninga/article/ view/1258/880

11 Moreira, LR, Dumith SC, Paludo SS. Condom use in last sexual intercourse among undergraduate students: how many are using them and who are they? Ciênc. Saúde Coletiva [Internet]. 2018 [citado2019 out4];23(4):125566. Disponível em: http://www.scielo.br/ scielo.php?script=sci_arttext\&pid=S1413$81232018000401255 \& \overline{l n} \mathrm{n}=\mathrm{en}$

12 Borba CR, Cardoso MV, Nagano SYM, Moraes FRR. Perfil do uso de métodos anticoncepcionais entre as estudantes dos cursos da área da saúde na Universidade Federal do Tocantins do campus universitário de Palmas. Rev. Amazônia Science e Health. [Internet]. 2017 [citado $2019 \mathrm{dez}$ 19]; 5(2):814. Disponível em: http://ojs.unirg.edu.br/ index.php/2/article/view/1641/pdf

13 Ajayi AI, Ismail KO, Akpan W. Factors associated with consistent condom use: a crosssectional survey of two Nigerian universities. BMC Public Health. [Internet]. 2019 [citado 2019 out 29]; 19(1):1-11. doi: 10.1186/s12889019-7543-1.

14 Brandão ER. Métodos contraceptivos reversíveis de longa duração no Sistema Único de Saúde: o debate sobre a (in) disciplina da mulher. Ciênc. Saúde Coletiva [Internet]. 2019 Mar [citado 2019 set 24]; 24(3):875-9. Disponível em: http://dx.d oi:101590/1413-81232018243.10932017

15 Higgins JA, Smith NK. The Sexual acceptability of contraception: reviewing the literature and building a new concept. J Sex
Res [Internet]. 2016 [cited 2018 Sep 2018]; 53(4-5): 417-56. Available from: http://dx.doi. org/10.1080/00224499.2015.1134425 